\title{
A GENERAL REPRESENTATION THEOREM FOR ANALYTIC SOLUTIONS OF FIRST-ORDER ALGEBRAIC DIFFERENTIAL EQUATIONS IN SECTORS
}

\author{
BY
}

\author{
STEVEN B. BANK
}

ABSTRACT. In this paper, we obtain precise asymptotic representations for a broad class of solutions of first-order algebraic differential equations whose coefficients belong to a certain type of function field.

1. Introduction. This paper completes an investigation into the representation of solutions of first-order algebraic differential equations which was begun in [1] and continued in [2].

The class of equations that we treat consists of first-order differential equations $\Omega\left(z, y, y^{\prime}\right)=0$, where $\Omega$ is a polynomial in $y$ and $y^{\prime}$, whose coefficients belong to a certain type of function field which was introduced and investigated by W. Strodt in [9]. Such a field consists of functions, each defined and analytic in a sectorial region approximately of the form

$$
a<\arg \left(z-\beta e^{i(a+b) / 2}\right)<b
$$

(for fixed $a$ and $b$ in $(-\pi, \pi)$ and some $\beta \geq 0)$, and has the property that there is a fixed nonnegative integer $p$ (called the rank of the field) such that the field contains all logarithmic monomials of rank $\leq p$ (i.e. all functions of the form

$$
M(z)=K z^{a_{0}}(\log z)^{a_{1}}(\log \log z)^{a_{2}} \ldots\left(\log _{p} z\right)^{a_{p}}
$$

for real $a_{j}$ and complex $K \neq 0$ ); and, in addition, for every element $($ in the field except zero, there is a logarithmic monomial $M$ of rank $\leq p$ which is asymptotically equivalent to $f$ as $z \rightarrow \infty$ over a filter base (denoted $F(a, b)$ ) which consists essentially of the sectors (1) as $\beta \rightarrow+\infty$. (We point out that we are using here the stronger concepts of "asymptotically equivalent" $(\sim)$ and "smaller rate of growth" (《), which were introduced by Strodt in $[7, \S \S 13,17]$. For the reader's convenience, these concepts are reviewed in $\$ 2$ below.) Of course, the set of all rational combinations of logarithmic monomials of rank $\leq p$ is the simplest example of such a field.

Received by the editors September $17,1973$.

AMS (MOS) subject classifications (1970). Primary 34A 20, 34D05, 34E05.

Key words and phrases. Algebraic differential equations, analytic solutions, representation of solutions, asymptotic behavior of solutions. 
In [4], [7] and [10], first-order equations, $\Omega\left(z, y, y^{\prime}\right)=0$, whose coefficients belong to such fields of arbitrary rank $p$, were treated and existence theorems were proved for solutions which are themselves asymptotically equivalent to logarithmic monomials over $F(a, b)$. In [3, p. 132], existence theorems were proved for solutions of $\Omega\left(z, y, y^{\prime}\right)=0$ (again for arbitrary rank $p$ ), which are of larger rate of growth than all logarithmic monomials over $F(a, b)$, and for solutions which are of smaller rate of growth than all logarithmic monomials. (These solutions were of the form $\exp \int W$, where $W$ is a function which is asymptotically equivalent to a logarithmic monomial of rank $\leq p$.)

In [1] and [2], the converse problem of determining the form of arbitrary solutions of such first-order equations was investigated. In [1], it was shown for arbitrary rank $p$, that any meromorphic solution which is of larger rate of growth over $F(a, b)$ than a predetermined power of $z$ must be of the form $\exp \int M(z)(1+\epsilon(z))$, where $M$ is a logarithmic monomial of rank $\leq p$ and where the analytic function $\epsilon(z)$ tends to zero over $F(a, b)$. It was also shown that nonidentically zero solutions which are of smaller rate of growth than a predetermined power of $z$ are also of this form. In [2], we treated solutions of "intermediate" growth, in the special case when the rank of the coefficient field is zero. More specifically, we considered all solutions $y_{0}(z)$ which are defined and meromorphic in a sector of the form (1), and which are "comparable" with all logarithmic monomials $M$ of rank $\leq 1$ (in the sense that for any such $M$, one of the relations $y_{0} \ll M, M \ll y_{0}$ or $y_{0} \sim c M$ for some constant $c \neq 0$ is valid over $F(a, b)$ ). It was shown in [2], that when the rank of the coefficient field is zero, then any such solution, which is not of larger rate of growth than all powers of $z$, and is not of smaller rate of growth than all powers of $z$ over $F(a, b)$, must be asymptotically equivalent over $F(a, b)$ to a logarithmic monomial of rank $\leq 1$. In addition, it was shown that the analogous conclusion no longer holds when the coefficients belong to fields of rank higher than zero (i.e. when logarithms can actually appear in the coefficients). Thus the results of [1] and [2] provide an asymptotic representation for all solutions which are comparable with all monomials of rank $\leq 1$ over $F(a, b)$, in the case of first-order equations with coefficients in fields of rank zero.

In this paper, we return to the general case of first-order equations with coefficients in fields of arbitrary rank $p$, and in analogy with [1] and [2], we seek to determine the form of those nonidentically zero meromorphic solutions which are comparable with all logarithmic monomials of rank $\leq p+1$ over $F(a, b)$. (Such a solution is automatically nowhere zero on some element of $F(a, b)$ (see $\$ 7$ below).). However, as we will explain further below, the presence of logarithms in the coefficients forces us to impose a more stringent condition on the solutions 
$y_{0}(z)$ that we treat, namely that $y_{0}$ be "power-comparable" with all monomials $M$ of rank $\leq p+1$ (in the sense that for each such $M$ and each positive integer $n$, some branch of $y_{0}^{1 / n}$ is comparable with $M$ ). Our main result ( $\$ 3$ below) states that any such solution is either asymptotically equivalent to a logarithmic monomial of rank $\leq p+1$ over $F(a, b)$ or is of the form $\exp \int N(z)(1+\epsilon(z))$, where $N$ is a logarithmic monomial of rank $\leq p$ and the analytic function $f(z)$ tends to zero over $F(a, b)$.

The necessity for assuming the condition of "power-comparability" is indicated by the following facts: If $y_{0} \ll 1$ over $F(a, b)$, then for each positive integer $n, y_{0}^{1 / n}$ tends to zero over $F(a, b)$. From Cauchy's formula for derivatives (see [6, p. 309]), it follows that if an analytic function $f$ tends to zero over $F(a, b)$, then $z f^{\prime}(z)$ also tends to zero over $F(a, b)$, which suffices for the case when the rank of the coefficient field is zero (i.e. no logarithms). However, it is not necessarily true that $(z \log z) f^{\prime}(z)$ tends to zero over $F(a, b)$, which causes difficulty in the case when the rank of the coefficient field is higher than zero (e.g. let $f(z)=z^{i} / \log z$ where $i^{2}=-1$ ). But if $y_{0}$ is power-comparable with the constant function 1 , and $y_{0} \ll 1$, then $f=y_{0}^{1 / n}$ is $\ll<1$ for each positive integer $n$, and it follows from the properties of the relation " $<"$ " (see $[7, \S 28])$ that $\left(z \log z \cdots \log _{q} z\right) f^{\prime}(z)$ tends to zero over $F(a, b)$ for each positive integer $q$.

In $\$ 15$, we show that the solutions, whose existence was proved in [3], [4], [7] and [10], all possess the property of being power-comparable with all logarithmic monomials. In addition, it is shown that there are analytic functions which are power-comparable with all logarithmic monomials over $F(a, b)$, but which are neither asymptotically equivalent to a logarithmic monomial nor of the form $\exp \int N(z)(1+\epsilon(z))$ where $N$ is a logarithmic monomial and $\epsilon(z)$ tends to zero over $F(a, b)$. Also in $\$ 15$, we take the opportunity to correct three printer's errors in [2].

In $\$ 16$, is an appendix containing five lemmas which are needed several times in the paper. They are put at the end of the paper to avoid unduly interrupting the main line of thought.

2. Preliminaries. (a) $[7, \S 94]$. Let $-\pi \leq a<b \leq \pi$. For each nonnegative real-valued function $\psi$ on $(0,(b-a) / 2)$, let $T(\psi)$ be the union (over $\delta \epsilon$ $(0,(b-a) / 2))$ of all sectors

$$
a+\delta<\arg (z-\psi(\delta) \exp (i(a+b) / 2))<b-\delta .
$$

The set of all $T(\psi)$ (for all choices of $\psi$ ) is denoted $F(a, b)$ and is a filter base which converges to $\infty$ by $[7, \S 95]$. Each $T(\psi)$ is simply connected by $[7, \S 93]$. If $W(z)$ is analytic in $T(\psi)$, then the symbol $\int W$ will stand for a primitive of $W$ in $T(\psi)$. 
(b) Unless otherwise indicated, $\log z$ will denote the principal branch of the $\operatorname{logarithm}$ in $S:|\arg z|<\pi$. It is then easy to see that $\log (\log z)$ is defined and analytic for those points in $S$ where $|z|>1$. We denote this function by $\log _{2} z$. By induction, the function $\log _{q+1} z=\log \left(\log _{q} z\right)$ is defined and analytic for those points in $S$ where $|z|>e_{q}(0)$ (where $e_{q}(z)$ is the qth iterate of the exponential function). To complete the definition, $\log _{q} z$ is defined to be $z$ when $q=0$. A logarithmic monomial of rank $\leq p$ is a function of the form (2) (which, of course, is defined to be $\left.K\left(\exp \Sigma_{j=0}^{p} \alpha_{j} \log _{j+1} z\right)\right)$. Clearly, for any logarithmic monomial, there is an element of $F(a, b)$ on which it is defined and analytic.

(c) $[7, \S 11]$. If $f$ is meromorphic in an element of $F(a, b)$ and $\lambda$ is a complex number, then $f \rightarrow \lambda$ over $F(a, b)$ means that for any $\epsilon>0$, there is an element of $F(a, b)$ on which $|f(z)-\lambda|<\epsilon$. Similarly, $f \rightarrow \infty$ over $F(a, b)$ means that for any $A>0$, there is an element of $F(a, b)$ on which $|f(z)|>A$. If $f$ and $g$ are meromorphic in an element of $F(a, b)$ with $g \equiv 0$, we will occasionally use the notation $f=o(g)$ over $F(a, b)$ to mean $f / g \rightarrow 0$. Similarly, $f=O(g)$ over $F(a, b)$ will mean $\mathrm{f} / \mathrm{g}$ is bounded on some element of $F(a, b)$. From Cauchy's formula for derivatives, it follows $\left[6\right.$, p. 309] that, if $f \rightarrow 0$ over $F(a, b)$, then $z f^{\prime}(z) \rightarrow 0$ over $F(a, b)$.

(d) $[7, \S \S 13,17]$. If $f$ is analytic in an element of $F(a, b)$ then $f \ll 1$ over $F(a, b)$ means that in addition to $f \rightarrow 0$, for all positive integers $j$ and $k$ we have $\theta_{j}^{k} f \rightarrow 0$ where $\theta_{j} f=\left(z \log z \cdots \log _{j-1} z\right) f^{\prime}$ and where $\theta_{j}^{k}$ is the $k$ th iterate of the operator $\theta_{j}$. Then $f \ll g, f \gg g, f \sim g$ and $f \approx g$ over $F(a, b)$ mean, respectively, $f / g \ll 1, g / f \ll 1, f-g \ll g$ and finally $f \sim c g$ for some constant $c \neq 0$. The crucial property $[7, \S 28]$ of the relation " $<$ " is that if $f \ll 1$ over $F(a, b)$, then $\theta_{j} f \ll 1$ over $F(a, b)$ for all $j>0$. From this it easily follows that if $f \ll M$, where $M$ is a nonconstant logarithmic monomial of any rank, then $f^{\prime} \ll M^{\prime}$. A function $f$ is said to be comparable with a function $g$ over $F(a, b)$ if one of the relations $f<<g, f \approx g, f \gg g$ is valid over $F(a, b)$. A function $f$ is said to be power-comparable with a function $g$ over $F(a, b)$, if $f$ is nowhere zero on some element of $F(a, b)$ and if there is an analytic branch $L(z)$ of the logarithm of $f$ such that for every positive integer $n, \exp (L / n)$ is comparable with $g$ over $F(a, b)$. (Since two analytic branches of the logarithm of $f$ differ by a constant on an element of $F(a, b)$, it follows easily that the definition of power-comparable is independent of which branch is used.) Finally, if $b \sim M$ where $M$ is the monomial (2), then $\delta_{j}(b)$ will denote $\alpha_{j}$ for $j \leq p$ and will denote zero for $j>p$.

(e) $[9$, p. 244]. Let $p$ be a nonnegative integer. A logarithmic field of rank $p$ over $F(a, b)$ is a set $\Gamma$ of functions, each defined and meromorphic in an element of $F(a, b)$, with the following properties: (i) $\Gamma$ is a field (where, as usual, we identify two elements of $\Gamma$ if they agree on an element of $F(a, b)$ ); (ii) $\Gamma$ contains 
all logarithmic monomials of rank $\leq p$; and (iii) for every element $f$ in $\Gamma$ except zero, there exists a logarithmic monomial $M$ of rank $\leq p$ such that $f \sim M$ over $F(a, b)$.

3. We now state our main result. The proof will be completed in $\S 14$.

Theorem. Let $\Omega\left(z, y, y^{\prime}\right)=\Sigma_{i, j \geq 0} f_{i j}(z) y^{i}\left(y^{\prime}\right)^{j}$ be a polynomial in $y$ and $y^{\prime}$, whose coefficients $f_{i j}$ belong to a logarithmic field of rank $p$ over $F(a, b)$ for some nonnegative integer $p$, and let some coefficient be not identically zero. Let $y_{0}(z)$ be a function which is defined, meromorphic and not identically zero in an element of $F(a, b)$ and which satisfies $\Omega\left(z, y_{0}(z), y_{0}^{\prime}(z)\right) \equiv 0$. Then if $y_{0}$ is comparable with all logaritbmic monomials of rank $\leq 0$, there is an element of $F(a, b)$ on which $y_{0}$ is nowbere zero. In addition, if $y_{0}$ is power-comparable with all logaritbmic monomials of rank $\leq p+1$, then at least one of the following two conclusions must bold:

(A) There exists a logaritbmic monomial $M$ of rank $\leq p+1$ such that $y_{0} \sim M$ over $F(a, b)$.

(B) There exist, in an element of $F(a, b)$, an analytic function $V(z)$ and a logaritbmic monomial $N(z)$ of rank $\leq p$, such that $V(z)=N(z)(1+o(1))$ over $F(a, b)$ and $y_{0}=\exp \int V$.

4. Definition. Let $y_{0}(z)$ be a function which is defined and meromorphic in an element of $F(a, b)$ and let $p$ be a positive integer. Let $q$ be a nonnegative integer $\leq p$, let $a_{0}, a_{1}, \cdots, a_{q-1}$ be real numbers (if $q \geq 1$ ), and let $\alpha_{q}$ be either a real number, $+\infty$ or $-\infty$. We call the sequence $\left(a_{0}, a_{1}, \cdots, a_{q}\right)$, a $p$ exponent sequence for $y_{0}$ of length $q$ over $F(a, b)$, if one of the following five conditions holds over $F(a, b)$ :

(a) $q=p, a_{p}$ is a real number, and for any real number $\epsilon>0$, we have

$$
\left(\log _{p} z\right)^{-\epsilon} Q \ll y_{0} \ll\left(\log _{p} z\right)^{\epsilon} Q
$$

where

$$
Q(z)=z^{a_{0}}(\log z)^{a_{1}} \ldots\left(\log _{p} z\right)^{a_{p}} .
$$

(b) $q=0, a_{q}=+\infty$, and for every real number $a>0$ we have $y_{0} \gg z^{a}$.

(c) $q=0, a_{q}=-\infty$, and for every real number $a>0$ we have $y_{0} \ll z^{-a}$.

(d) $q \geq 1, a_{q}=+\infty$, and if we set,

$$
Q_{1}(z)=z^{a_{0}}(\log z)^{a_{1}} \ldots\left(\log _{q-1} z\right)^{a_{q-1}},
$$

then for every $\epsilon>0$, we have 


$$
\left(\log _{q-1} z\right)^{-\epsilon} Q_{1} \ll y_{0} \ll\left(\log _{q-1} z\right)^{\epsilon} Q_{1}
$$

and

$$
y_{0} \gg\left(\log _{q} z\right)^{\epsilon} Q_{1}
$$

(e) $q \geq 1, a_{q}=-\infty$, and if $Q_{1}$ is as in (6), then for every $\epsilon>0,(7)$ holds while (8) is replaced by

$$
y_{0} \ll\left(\log _{q} z\right)^{-\epsilon} Q_{1} .
$$

In Case (a) (i.e. where $a_{q}$ is a real number), we say that the sequence is logarithmic. In Cases (b)-(d) (i.e. where $a_{q}$ is either $+\infty$ or $-\infty$,) we say that the sequence is nonlogaritbmic.

5. Lemma A. Let $y_{0}(z)$ be a function which is defined and meromorpbic in an element of $F(a, b)$, and which is comparable over $F(a, b)$ with all logaritbmic monomials of rank $\leq p$, where $p$ is a positive integer. Then $y_{0}$ possesses a $p$ exponent sequence over $F(a, b)$ of some length $q$.

Proof. Let $A_{0}$ be the set of all real $a$ for which $y_{0} \ll z^{a}$ over $F(a, b)$. We distinguish three possibilities. If $A_{0}$ is empty, then by comparability, we must have $y_{0} \gg z^{a}$ for all $\alpha$. (Note that if $y_{0} \approx z^{a}$, then $a+1$ would belong to $A_{0}$.) Hence in this case, the sequence $(+\infty)$ is a p-exponent sequence for $y_{0}$. The second possibility is that $A_{0}$ is nonempty but unbounded from below. In this case, clearly $y_{0} \ll z^{\alpha}$ for all $\alpha$, so $(-\infty)$ is a p-exponent sequence. The last possibility for $A_{0}$ is that it is nonempty and bounded from below. By comparability it follows that if $\alpha_{0}$ is the infimum of $A_{0}$, then for any $\epsilon>0$,

$$
z^{a_{0}-\epsilon} \ll y_{0} \ll z^{a_{0}+\epsilon}
$$

We now let $A_{1}$ be the set of all real $a$ for which $y_{0} \ll z^{a}{ }^{0}(\log z)^{a}$. Again we consider the three possibilities for $A_{1}$. Since $p \geq 1$, it follows from comparability and (10) that if $A_{1}$ is empty, then $\left(\alpha_{0},+\infty\right)$ is a $p$-exponent sequence for $y_{0}$. Similarly, if $A_{1}$ is nonempty and unbounded from below, then $\left(a_{0},-\infty\right)$ is a $p$ exponent sequence for $y_{0}$. In the last possibility for $A_{1}$, if $a_{1}$ is the infimum of $A_{1}$, then for any $\epsilon>0$,

$$
z^{a_{0}}(\log z)^{a_{1}-\epsilon} \ll y_{0} \ll z^{a_{0}}(\log z)^{a_{1}+\epsilon} .
$$

If $p=1$, then (11) shows that $\left(a_{0}, a_{1}\right)$ is a pexponent sequence for $y_{0}$. If $p>1$, we consider the set $A_{2}$ of all real $a$ for which $y_{0}<z^{a}{ }^{a}(\log z)^{a}\left(\log _{2} z\right)^{a}$, and again consider the above three possibilities for $A_{2}$. Continuing this way, it is easy to see that $y_{0}$ possesses a $p$-exponent sequence. 
6. General hypothesis (for $\$ \S 7-13)$. $p$ is a positive integer; $y_{0}(z)$ is a function which is defined, meromorphic, and not identically zero in an element of $F(a, b) ; y_{0}(z)$ is comparable with all logarithmic monomials of rank $\leq 0 ; y_{0}(z)$ is a solution of a first-order differential equation $\Omega\left(z, y, y^{\prime}\right)=0$, where $\Omega$ is a polynomial in $y$ and $y^{\prime}$, whose coefficients belong to a logarithmic field of rank $p$ over $F(a, b)$ and where some coefficient of $\Omega$ is not identically zero.

7. Lemma B. Assume \$6. Then there exists an element of $F(a, b)$ on which $y_{0}$ is nowbere zero.

Proof. If $y_{0} \ll z^{a}$ over $F(a, b)$ for all real $a$, then the conclusion follows from $[1, \S 4]$. In the contrary case, it follows from comparability that $y_{0} \gg z^{a_{0}}$ for some real $a_{0}$, and in this case the conclusion is obvious.

Remark. Since $[1, \$ 4]$ is valid for all nonnegative integers $p$, it follows that Lemma $B$ holds even if $p=0$.

8. Lemma C. Assume $\$ 6$, and in addition, assume that $y_{0}$ is power-comparable with all logarithmic monomials of rank $\leq p$ over $F(a, b)$. Then if $y_{0}$ pos. sesses a nonlogaritbmic p-exponent sequence of length $\geq 1$ over $F(a, b)$, whose last coordinate is $+\infty$, then there exist, in an element of $F(a, b)$, an analytic function $V(z)$ and a logarithmic monomial $N(z)$ of rank $\leq p$, sucb that $V(z)=$ $N(z)(1+o(1))$ over $F(a, b)$ and $y_{0}=\exp \int V$.

Proof. Let $\left(\alpha_{0}, \cdots, \alpha_{q}\right)$ be the $p$-exponent sequence for $y_{0}$, with $q \geq 1$ and $\alpha_{q}=+\infty$. Then if $Q_{1}$ is given by (6), we have that for every $\epsilon>0,(7)$ and (8) hold. Now define $w_{0}=y_{0} / Q_{1}$. Thus from (7) and (8), we have that for every $\epsilon>0$,

$$
\left(\log _{q-1} z\right)^{-\epsilon} \ll w_{0} \ll\left(\log _{q-1} z\right)^{\epsilon} \text { over } F(a, b)
$$

and

$$
w_{0} \gg\left(\log _{q} z\right)^{\epsilon} \text { over } F(a, b) \text {. }
$$

In view of (12), $w_{0}$ is analytic in an element of $F(a, b)$. By $\$ 6, y_{0}$ is a solution of the first-order equation $\Omega\left(z, y, y^{\prime}\right)=0$. Since $q \leq p$, it follows easily that $w_{0}$ is a solution of an equation,

$$
\Lambda\left(z, w, w^{\prime}\right)=0
$$

where

$$
\Lambda\left(z, w, w^{\prime}\right)=\sum g_{k j}(z) w^{k}\left(w^{\prime}\right)^{j}
$$

is a polynomial in $w$ and $w^{\prime}$ whose coefficients belong to the same field as the 
coefficients of $\Omega$, and some coefficient of $\Lambda$ is not identically zero by $[7, \S 73]$. Hence if $g_{k j} \equiv 0$, then by $\$ 2(e)$, we have

$$
g_{k j}=c_{k j} z^{a_{k j 0}}(\log z)^{a_{k j 1}} \ldots\left(\log _{p} z\right)^{a_{k j p}}\left(1+E_{k j}\right),
$$

where $c_{k j}$ is a nonzero constant, where $a_{k j 0}, \cdots, a_{k j p}$ are real numbers and where the $E_{k j}$ are elements of the field which are $<1$ over $F(a, b)$. Let $J$ be the set of all $(k, j)$ for which $g_{k j} \equiv 0$. We define real numbers $d_{0}, \cdots, d_{p}$ and sets $J_{0}, \cdots, J_{p}$ inductively as follows. Let

$$
\begin{gathered}
d_{0}=\max \left\{a_{k j 0}-j:(k, j) \in J\right\} \\
J_{0}=\left\{(k, j):(k, j) \in J \text { and } a_{k j 0}-j=d_{0}\right\}
\end{gathered}
$$

Assuming that $d_{0}, \cdots, d_{m-1}$ and $J_{0}, \cdots, J_{m-1}$ have been defined, where $1 \leq$ $m \leq p$, let

$$
\begin{gathered}
d_{m}=\max \left\{a_{k j m}-j:(k, j) \in J_{m-1}\right\} \\
J_{m}=\left\{(k, j):(k, j) \in J_{m-1} \text { and } a_{k j m}-j=d_{m}\right\}
\end{gathered}
$$

If $k$ is a nonnegative integer, then by (12), we have

$$
w_{0}^{k}=O\left(\left(\log _{q-1} z\right)^{k \epsilon}\right) \text { over } F(a, b) \text { for all } \epsilon>0,
$$

and if $j$ is a nonnegative integer then by (12) and $\S_{2}(d)$, we have for every $\epsilon>0$,

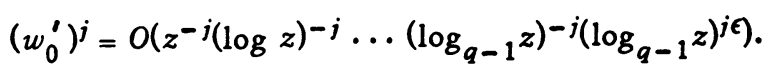

Now set,

$$
\phi(z)=z^{d_{0}}(\log z)^{d_{1}} \ldots\left(\log _{q-2} z\right)^{d_{q-2}} \text { if } q \geq 2
$$

while if $q=1$, set $\phi(z) \equiv 1$.

We now consider the set $J-J_{q-1}$, and we distinguish two possibilities. For those $(k, j)$ which belong to every set $J_{r}$ where $0 \leq r \leq q-2$, but not to $J_{q-1}$, we have $a_{k j r}-j=d_{r}$ for $0 \leq r \leq q-2$, but $\epsilon_{k j}=d_{q-1}-\left(a_{k j, q-1}-j\right)>0$. Letting $\epsilon_{1}$ be a fixed positive number which is less than all of the numbers $\epsilon_{k j} / 2$, and choosing the arbitrary number $\epsilon>0$ in (21) and (22) so that $(k+j) \epsilon$ $\left\langle\epsilon_{1}\right.$ for all these $(k, j)$, it follows from (16), (21), (22), that for these $(k, j)$,

$$
g_{k j} w_{0}^{k}\left(w_{0}^{\prime}\right)^{j}=O\left(\left(\log _{q-1} z\right)^{d} q-1^{-\epsilon_{1}} \phi(z)\right) \text { over } F(a, b),
$$


where $\phi(z)$ is as in (23). For those $(k, j)$ which fail to belong to some set $J_{r}$, where $0 \leq r \leq q-2$, it follows easily from (16), (21) and (22), that for some $t$, $0 \leq t \leq q-2$, the left side of (24) is

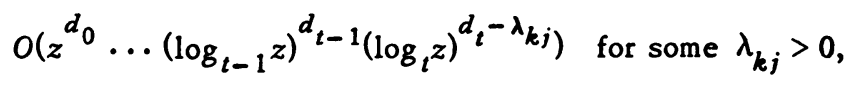

and hence (24) holds for these $(k, j)$ also. Thus $(24)$ is valid for all $(k, j)$ in $J-J_{q-1}$, and hence, since $w_{0}$ is a solution of equation (14), we have

$$
\sum\left\{g_{k j} w_{0}^{k}\left(w_{0}^{\prime}\right)^{j}:(k, j) \in J_{q-1}\right\}=O(H(z)),
$$

where $H(z)=\left(\log _{q-1} z\right)^{d}{ }_{q-1}^{-\epsilon}{ }^{-\epsilon} \phi(z)$. (We remark here, for future use, that although both (12) and (13) are available to us, (13) was not used in developing (25).)

Now let $r=\max \left\{k+j:(k, j) \in J_{q-1}\right\}$ and let $I$ be the set of all $(k, j)$ in $J_{q-1}$ such that $k+j=r$. Isolating the terms of $I$ in (25), and dividing by $w_{0}^{r}$ (which is permissible by (12)), we obtain

$$
\sum_{(k, j) \in I} g_{k j}\left(w_{0}^{\prime} / w_{0}\right)^{j}=H_{1}(z)+O(H(z)) / w_{0}^{r},
$$

where $H_{1}(z)=-\Sigma\left\{g_{k j}\left(w_{0}^{\prime}\right)^{j} / w_{0}^{r-k}:(k, j) \in J_{q-1}-l\right\}$. Now since $y_{0}$ is powercomparable with all logarithmic monomials of rank $\leq p$, it is easily seen that the same is true of $w_{0}$. Since $j<r-k$ for each term in $H_{1}(z)$, it now follows easily from (13), (16), the definitions of $J_{q-1}$ and $H$, and Appendix Lemma 5, that for every $a>0$, the right side of (26) is $O\left(\left(\log _{q} z\right)^{-a}\left(\log _{q-1} z\right)^{d} q-1 \phi(z)\right)$, where $\phi$ is as in (23). Again using (16) and the definition of $J_{q-1}$, and dividing (26) by $\left(\log _{q-1} z\right)^{d} q-1 \phi(z)$, we obtain from (26) that

$$
\sum_{(k, j) \epsilon I} G_{k j} v_{0}^{j}=O\left(\left(\log _{q} z\right)^{-a}\right) \text { for all } a>0,
$$

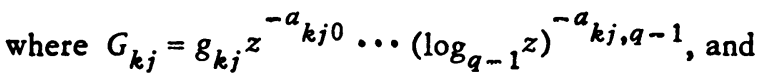

$$
v_{0}=z(\log z) \ldots\left(\log _{q-1} z\right) w_{0}^{\prime} / w_{0} \text {. }
$$

We now consider the algebraic polynomial,

$$
\Phi(v)=\sum_{(k, j) \in l} G_{k j} v^{j}
$$

The coefficients $G_{k j}$ are nonzero functions which clearly belong to the same logarithmic field as do the $g_{k j}$, and clearly $\delta_{m}\left(G_{k j}\right)=0$ for $m<q$, while for $m \geq q, \delta_{m}\left(G_{k j}\right)=\delta_{m}\left(g_{k j}\right)$ (see $\oint_{2}(\mathrm{~d})$ ). Let $\beta$ and $\sigma$ be, respectively, the minimum 
and maximum of the set $\{j:(k, j) \in I\}$. Then $\Phi$ is a polynomial of degree $\sigma$; and $\sigma>0$, for otherwise by (27), $G_{r 0}$ would be $O\left(\left(\log _{q} z\right)^{-\alpha}\right)$ for all $\alpha>0$, which contradicts the obvious relation $G_{r 0} \gg\left(\log _{q} z\right)^{a} r 0 q^{-1}$. Thus $\Phi$ is an algebraic polynomial of positive degree $\sigma$ with coefficients in a logarithmic field of rank $p$. It follows from [9, Theorem II, p. 244] (by applying this result to, in the terminology of $[9, \mathrm{p} .246]$, the logarithmic quadruple $\left(F, E_{0}^{*}(F), R, s_{p}\right)$, where $F=F(a, b)$ and $R$ is the set of real numbers), that there exists a logarithmic field of rank $p$ over $F(a, b)$ in which $\Phi(v)$ factors completely. Hence there exist distinct functions $B_{1}, \cdots, B_{t}$, each defined and meromorphic in an element $T$ of $F(a, b)$ such that,

$$
\Phi(v)=G_{r-\sigma, \sigma} v^{\beta}\left(v-B_{1}(z)^{m_{1}} \cdots\left(v-B_{t}(z)\right)^{m_{t}}\right.
$$

for all meromorphic functions $v=v(z)$ defined on $T$, where $m_{1}, \cdots, m_{t}$ are positive integers such that $\Sigma_{m_{j}}=\sigma-\beta$. (Of course, if $\sigma=\beta$, then the set $\left\{B_{1}, \cdots, B_{t}\right\}$ is empty.) Furthermore, if $1 \leq j \leq t$ and $1 \leq i \leq t$ and $i \neq j$ then by $\S 2(e)$ there exist logarithmic monomials $M_{j}$ and $M_{i j}$ of rank $\leq p$ such that

$$
B_{j} \sim M_{j} \text { and } B_{i}-B_{j} \sim M_{i j} \quad \text { over } F(a, b) \text {. }
$$

Since the $B_{j}$ are roots of $\Phi$, it follows from either $[9, \S 36]$ or $[4, \S 5]$ that the $M_{j}$ can be constructed using the algorithms in $[9, \S 28]$ or $[4, \$ 26]$. Since $\delta_{m}\left(G_{k j}\right)=$ 0 for $m<q$, these algorithms show that for $1 \leq j \leq t$,

$$
\delta_{m}\left(B_{j}\right)=\delta_{m}\left(M_{j}\right)=0 \text { for } m<q .
$$

Let $A_{0}$ be the set of all pairs $(i, j)$ of integers in $\{1, \cdots, t\}$, with $i \neq j$. Let $A$ be the subset of $A_{0}$ consisting of those $(i, j)$ for which $\delta_{m}\left(M_{i j}\right)=0$ for $m<q$. Hence clearly we can choose $\lambda>2$ so large that $\left(\log _{q} z\right)^{\lambda} B_{j} \rightarrow \infty$ for each $j$, and $\left(\log _{q} z\right)^{\lambda}\left(B_{i}-B_{j}\right) \rightarrow \infty$ for $(i, j) \in A$. If $(i, j) \in A_{0}-A$, and $k$ is the smallest nonnegative integer for which $\delta_{k}\left(M_{i j}\right) \neq 0$, then we must have $\delta_{k}\left(M_{i j}\right)<0$, for otherwise $M_{i j} \gg M_{i}$ and $M_{i j} \gg M_{j}$ (by (32)), which would obviously contradict (31). Thus for $(i, j) \in A_{0}-A$, we have $\left(\log _{q} z\right)^{\lambda}\left(B_{i}-B_{j}\right) \rightarrow 0$. Thus we can choose an element $T_{1}$ in $F(a, b)$ on which

$$
\begin{gathered}
\left|\left(\log _{q} z\right)^{\lambda} B_{j}(z)\right|>2 \text { for } j=1, \ldots, t, \\
\left|\left(\log _{q} z\right)^{\lambda}\left(B_{i}(z)-B_{j}(z)\right)\right|>2 \text { for }(i, j) \in A, \\
\left|\left(\log _{q} z\right)^{\lambda}\left(B_{i}(z)-B_{j}(z)\right)\right|<1 / 4 \text { for }(i, j) \in A_{0}-A .
\end{gathered}
$$


Now let $u_{0}=\left(\log _{q} z\right)^{\lambda} v_{0}, B_{0} \equiv 0$ and, for $0 \leq j \leq t$, let $W_{j}=\left(\log _{q} z\right)^{\lambda} B_{j}$. Now since $G_{r-\sigma, \sigma} \gg\left(\log _{q} z\right)^{d}$ where $d=a_{r-\sigma, \sigma, q}-1$, it follows directly from (27) (for a sufficiently large choice of $\alpha$ ) and (30), that there is an element $T_{2}$ in $F(a, b)$, contained in $T_{1}$, on which

$$
\left|u_{0}(z)-W_{0}(z)\right|^{\beta}\left|u_{0}(z)-W_{1}(z)\right|^{m_{1}} \cdots\left|u_{0}(z)-W_{t}(z)\right|^{m}<(1 / 2)^{\sigma} .
$$

Now let $z_{1}$ be a fixed element of $T_{2}$. Since $\beta+\Sigma m_{j}=\sigma$, it follows from (36) that it is impossible that $\left|u_{0}\left(z_{1}\right)-W_{j}\left(z_{1}\right)\right| \geq 1 / 2$ for each $j=0,1, \cdots, t$. Thus, there exists an index $m \in\{0,1, \cdots, t\}$ ( $m$ depending on $z_{1}$ ) such that,

$$
\left|u_{0}\left(z_{1}\right)-W_{m}\left(z_{1}\right)\right|<1 / 2 \text {. }
$$

We assert that, for this index $m$, we have

$$
\left|u_{0}(z)-W_{m}(z)\right| \leq 1 \text { for all } z \text { in } T_{2} .
$$

To prove (38), we assume that (38) fails for some point $z_{2}$ in $T_{2}$. Since there is a curve in $T_{2}$ joining $z_{1}$ and $z_{2}$, it follows from (37) and the continuity of $\left|u_{0}(z)-W_{m}(z)\right|$, that for some point $z_{3}$ in $T_{2}$ we have

$$
\left|u_{0}\left(z_{3}\right)-W_{m}\left(z_{3}\right)\right|=1 \text {. }
$$

But as above, from (36), there is an index $k$, such that

$$
\left|u_{0}\left(z_{3}\right)-W_{k}\left(z_{3}\right)\right|<1 / 2 .
$$

Clearly from (39) and (40), $k \neq m$ and

$$
\left|W_{m}\left(z_{3}\right)-W_{k}\left(z_{3}\right)\right| \leq 3 / 2 .
$$

We distinguish three cases. If neither $k$ nor $m$ is zero, then by (34) and (41), clearly $(k, m) \in A_{0}-A$. But from (39) and (40), we obtain $\left|W_{m}\left(z_{3}\right)-W_{k}\left(z_{3}\right)\right| \geq$ $1 / 2$ contradicting (35). The second case is $k=0$. Thus $W_{k}\left(z_{3}\right)=0$, so from (41), $\left|W_{m}\left(z_{3}\right)\right| \leq 3 / 2$ which contradicts (33) since $m \neq 0$. The final case is $m=0$, so from (41), it would follow that $\left|W_{k}\left(z_{3}\right)\right| \leq 3 / 2$ again contradicting (33). Thus (38) has been proved. From (38) (and (28)), we see that for all $z \in T_{2}$,

$$
\left|\left(w_{0}^{\prime}(z) / w_{0}(z)\right)-E(z)\right| \leq|z|^{-1} \cdots\left|\log _{q-1} z\right|^{-1}\left|\log _{q} z\right|^{-\lambda},
$$

where

$$
E(z)=z^{-1}(\log z)^{-1} \cdots\left(\log _{q-1} z\right)^{-1} B_{m} .
$$

We assert that

$$
m \neq 0 \text {. }
$$


To prove (44), we assume the contrary. Then $E(z) \equiv 0$. Letting $L(z)$ be a branch of $\log w_{0}$ on $T_{2}$, and noting that $\lambda>2$, it follows from (42) and Appendix Lemma 2 that if $z_{0}$ is a fixed point on $\arg z=(a+b) / 2$, lying in $T_{2}$ and of sufficiently large modulus, then as a function of $R, L\left(R z_{0}\right)$ is bounded on $[1,+\infty)$. Hence $w_{0}\left(R z_{0}\right)$ is bounded on $[1,+\infty)$ which clearly contradicts (13). Thus (44) holds.

We now set

$$
U=\left(w_{0}^{\prime} / w_{0}\right)-E
$$

Since $\lambda$ was chosen so that $\left(\log _{q} z\right)^{\lambda} B_{m} \rightarrow \infty$, it follows from (42) and (43) that $U / E \rightarrow 0$. Thus, from (45),

$$
w_{0}^{\prime} / w_{0}=E(1+o(1))
$$

In view of (43) and (31), we thus have

$$
w_{0}^{\prime} / w_{0}=z^{-1}(\log z)^{-1} \ldots\left(\log _{q-1} z\right)^{-1} M_{m}(1+o(1)) .
$$

From the definition of $w_{0}$ we have

$$
y_{0}^{\prime} / y_{0}=\left(w_{0}^{\prime} / w_{0}\right)+\left(Q_{1}^{\prime} / Q_{1}\right)
$$

where $Q_{1}$ is given by (6). If in (6), $a_{j}=0$ for $0 \leq j \leq q-1$, then $Q_{1}^{\prime} \equiv 0$ and since $M_{m}$ is a logarithmic monomial, the conclusion of the lemma now follows from (48) and (47). If not all $\alpha_{j}$ are zero in (6), let $n=\min \left\{j: \alpha_{j} \neq 0\right\}$. Then,

$$
Q_{1}^{\prime} / Q_{1} \sim a_{n} z^{-1} \ldots\left(\log _{n} z\right)^{-1}
$$

If $n<q-1$, then since $\delta_{j}\left(M_{m}\right)=0$ for $j<q$ by (32), clearly from (47), (48) and (49), we obtain $y_{0}^{\prime} / y_{0}=N(1+o(1))$, where $N$ is the monomial on the right side of (49), and hence we obtain the conclusion of the lemma. We are thus left with the case $n=q-1$. If $M_{m} \gg 1$, then by (47), (48) and (49), we obtain $y_{0}^{\prime} / y_{0}=$ $N_{1}(1+o(1))$, where $N_{1}=z^{-1} \cdots\left(\log _{q-1} z\right)^{-1} M_{m}$, and again we have the conclusion of the lemma. Similarly, if $M_{m}<1$, then we obtain $y_{0}^{\prime} / y_{0}=N(1+o(1))$, where $N$ is the monomial on the right of (49) with $n=q-1$, and again the lemma is proved. The final possibility for $M_{m}$ is that $M_{m} \approx 1$. Thus $M_{m}=c$ for some constant $c \neq 0$. Since $n=q-1$, we obtain from (47), (48), (49),

$$
y_{0}^{\prime} / y_{0}=z^{-1} \ldots\left(\log _{q-1} z\right)^{-1}\left(c+\alpha_{q-1}+o(1)\right) .
$$

Thus if $c+a_{q-1} \neq 0$, we obtain the conclusion of the lemma. We now show that $c+a_{q-1} \neq 0$, and so the proof of the lemma will be complete. We assume the contrary so $c=-a_{q-1}$. Since $a_{q-1}$ is a nonzero real number, so is $c$. If $c<0$, it follows directly from the representation $(47)$ and $[7, \S 103]$ that for all real $\alpha$, 
$\left(\log _{q} z\right)^{a} w_{0} \rightarrow 0$ over $F(a, b)$. This clearly contradicts (13). The remaining possibility is that $c>0$. In this case, we have by (12) that $w_{0}<<\left(\log _{q-1} z\right)^{c / 2}$. We set, $\psi=\left(\log _{q-1} z\right)^{-c / 2} w_{0}$, so $\psi \rightarrow 0$, and in view of (47), we have $\psi-$ $\left(\psi^{\prime} / W\right)=0$, where

$$
W=(c / 2) z^{-1} \cdots\left(\log _{q-1} z\right)^{-1}(1+o(1)) .
$$

Since $\psi \rightarrow 0$ and $w_{0} \rightarrow \infty$ (by (13)), there exists an element $T_{3}$ in $F(a, b)$ on which $0<|\psi|<1$ and $W$ is nowhere zero. Clearly (see $\$ 2(a)), T_{3}$ contains a closed sector, $\phi_{0}-\delta \leq \arg \left(z-x_{0}\right) \leq \phi_{0}+\delta$, where $\phi_{0}=(a+b) / 2, \delta=(b-a) / 4$ and $\arg x_{0}=\phi_{0}$. But since $c>0$, it follows from $[8, \S 73(b)]$ that the only function which is analytic and bounded on such a sector and satisfies the equation $y-\left(y^{\prime} / W\right)=0$, is the zero function. This contradicts the existence of $\psi$, so the case $c>0$ is also impossible and the proof of the lemma is now complete.

9. Lemma D. Assume $\$ 6$, and in addition, assume that $y_{0}$ is power-comparable with all logarithmic monomials of rank $\leq p$ over $F(a, b)$. Then if $y_{0}$ possesses a nonlogarithmic p-exponent sequence of length $\geq 1$ over $F(a, b)$, then there exist in an element of $F(a, b)$, an analytic function $V(z)$ and a logaritbmic monomial $N(z)$ of rank $\leq p$, sucb that $V(z)=N(z)(1+o(1))$ over $F(a, b)$ and $y_{0}=$ $\exp \int V$.

Proof. Let $\left(a_{0}, \cdots, \alpha_{q}\right)$ be the p-exponent sequence for $y_{0}$. If $a_{q}=+\infty$, the result follows from Lemma C. If $a_{q}=-\infty$, we set $u_{0}=1 / y_{0}$ (which is analytic by Lemma $\mathrm{B}$ ). Then it is easy to see that $u_{0}$ satisfies the hypothesis of Lemma $C$ having $p$-exponent sequence $\left(-\alpha_{0}, \cdots,-\alpha_{q}\right)$. Applying Lemma $C$ to $u_{0}$, the conclusion immediately follows for $y_{0}$.

10. General hypothesis (for $\$ \$ 11-13$ ). In addition to the hypothesis of $\$ 6$, we further assume that $y_{0}$ is power-comparable with all logarithmic monomials of rank $\leq p+1$, and that $y_{0}$ possesses a logarithmic p-exponent sequence $\left(a_{0}, \cdots, a_{p}\right)$. Let $Q$ be as in (5) and let $w_{0}=y_{0} / Q$. Let $\theta$ be the operator $\theta_{p+1}$ defined in $\$ 2(d)$.

11. Lemma E. Assume $\$ 10$. Then: (a) There exist $\epsilon_{2}>0$, a finite nonempty set $D$ of ordered pairs of nonnegative integers, and a nonzero constant $c_{k j}$ for each $(k, j) \in D$, such that

$$
\sum_{(k, j) \in D} c_{k j} w_{0}^{k}\left(\theta w_{0}\right)^{j}=O\left(\left(\log _{p} z\right)^{-\epsilon_{2}}\right) \quad \text { over } F(a, b) .
$$

(b) It is impossible that $w_{0} \gg\left(\log _{p+1} z\right)^{a}$ for all $\alpha>0$.

Proof. In view of $\$ 6$, it is clear that $w_{0}$ is a solution of an equation (14), 
whose coefficients are given by (16). Furthermore, since (4) holds for $y_{0}$, clearly (12) holds for $q=p+1$. Thus, defining $d_{0}, \cdots, d_{p}, J_{0}, \cdots, J_{p}$ as in (17)-(20), it follows that (25) holds when $q-1=p$. Since $a_{k j m}-j=d_{m}$ for $0 \leq m \leq p$ if $(k, j) \in J_{p}$, it follows from $(25)$ for $q-1=p$, that

$$
\sum_{(k, j) \epsilon J_{p}} c_{k j}\left(1+E_{k j}\right) w_{0}^{k}\left(\theta w_{0}\right)^{j}=O\left(\left(\log _{p} z\right)^{-\epsilon} 1\right) .
$$

Since the $E_{k j}$ belong to a logarithmic field of rank $p$, and are $\ll 1$, it follows from (iii) of $\$ 2(e)$ that for some $\delta>0, E_{k j} \ll\left(\log _{p} z\right)^{-\delta}$ for all $(k, j) \in J_{p}$. Let $\epsilon_{2}=\min \left\{\epsilon_{1}, \delta / 2\right\}$. Since (12) holds for $q-1=p$, so do (21) and (22). Choosing the arbitrary $\epsilon>0$ in (21) and (22) so that $(k+j) \epsilon<\delta-\epsilon_{2}$ for all $(k, j) \epsilon J_{p}$, it follows that $E_{k j} w_{0}^{k}\left(\theta w_{0}\right)^{j}=O\left(\left(\log _{p} z\right)^{-\epsilon}\right)$ and so (51) follows from (52) taking $D=J_{p}$

To prove (b), we assume the contrary, i.e. $w_{0} \gg\left(\log _{p+1} z\right)^{a}$ for all $a>0$. Let $n=\max \{k+j:(k, j) \in D\}$ and let $I$ be the set of $(k, j)$ in $D$ with $k+j=n$. Since $y_{0}$ is power-comparable with all monomials of rank $\leq p+1$, the same is clearly true of $w_{0}$. Thus if in (51), we divide by $w_{0}^{n}$ and isolate the terms of $l$, it follows from Appendix Lemma 5 and our assumption about $w_{0}$, that for all $a>0$,

$$
\sum_{(k, j) \epsilon !} c_{k j} v_{0}^{j}=o\left(\left(\log _{p+1} z\right)^{-a}\right), \text { where } v_{0}=\theta w_{0} / w_{0} .
$$

The left side of (53) is a polynomial (with constant coefficients) in $v_{0}$. If $m$ is the degree of this polynomial, then clearly $m>0$, and in view of (53) for $a=$ $2 m+1$, it follows from Appendix Lemma 1 (using $\left.\phi(z)=\left(\log _{p+1} z\right)^{-(2 m+1)}\right)$, that there is a complex root $\lambda$ such that $v_{0}-\lambda=O\left(\left(\log _{p+1} z\right)^{-2}\right)$. Let $\lambda=c+i d$ where $c$ and $d$ are real, let $L$ be a branch of $\log w_{0}$ and let $U=L-\lambda\left(\log _{p+1} z\right)$. From Appendix Lemma 2, it follows that if $z_{0}$ is a fixed point with $\arg z_{0}=(a+b) / 2$, of sufficiently large modulus, then for $R \geq 1$,

$$
\left|U\left(R z_{0}\right)-U\left(z_{0}\right)\right| \leq K\left(\log _{p+1}\left|z_{0}\right|\right)^{-1}
$$

where $K$ is a positive constant independent of $R$. Thus as functions of $R, U\left(R z_{0}\right)$ and hence $\exp \left(U\left(R z_{0}\right)\right)$ are bounded on $[1,+\infty)$. Since by assumption, $\left|w_{0}\left(R z_{0}\right)\right| \rightarrow$ $+\infty$ as $R \rightarrow+\infty$, it easily follows that we must have $c>0$. Now if we set $V=-U$, then by (54), $V\left(R z_{0}\right)$ and hence $\exp \left(V\left(R z_{0}\right)\right)$ are bounded functions of $R$ on $[1,+\infty)$. From this it follows that for some constant $K_{1}>0$, we have

$$
K_{1}\left|\log _{p}\left(R z_{0}\right)\right|^{c} \leq\left|w_{0}\left(R z_{0}\right)\right| \text { for all } R \geq 1 .
$$

But since (12) holds for $q-1=p$, and since $c>0$, we have $w_{0} \ll\left(\log _{p} z\right)^{c / 2}$ 
which clearly contradicts (55). This proves part (b).

12. Lemma F. Assume $\$ 10$. Then there exists a real number $\beta$, such that over $F(a, b)$,

$$
\left(\log _{p+1} z\right)^{\beta-\epsilon} \ll w_{0} \ll\left(\log _{p+1} z\right)^{\beta+\epsilon} \quad \text { for all } \epsilon>0 .
$$

Proof. Let $u_{0}=1 / y_{0}$. Then $u_{0}$ is analytic over $F(a, b)$ by Lemma $\mathrm{B}$, and it is easy to see that $u_{0}$ satisfies $\$ 10$ with p-exponent sequence $\left(-\alpha_{0}, \cdots\right.$, $\left.-a_{p}\right)$. Thus if $v_{0}=Q u_{0}$ (where $Q$ is as in (5)), we have by Lemma E, part (b), that it is impossible that $v_{0} \gg\left(\log _{p+1} z\right)^{a}$ for all $a>0$, in addition to it being impossible that $w_{0} \gg\left(\log _{p+1} z\right)^{\alpha}$ for all $\alpha>0$. But clearly $v_{0}=1 / w_{0}$, so by the comparability of $w_{0}$ with all monomials of rank $\leq p+1$, it follows that the set of all real $\alpha$ for which $w_{0} \ll\left(\log _{p+1} z\right)^{a}$, is nonempty and bounded from below, and (56) holds if $\beta$ is the infimum of this set.

13. Lemma G. Assume $\$ 10$. Then there exists a logarithmic monomial $M$ of rank $\leq p+1$ such that $y_{0} \sim M$ over $F(a, b)$.

Proof. We assume the contrary. Then clearly for every monomial $M$ of rank $\leq p+1$, we must have

$$
w_{0} / M \rightarrow 0 \text { or } \infty \text { over } F(a, b) \text {. }
$$

(If (57) failed for some $M$, then $w_{0} \ll M$ and $w_{0} \gg M$ are both false, so by comparability, $w_{0} \approx M$ and hence, $y_{0} \sim c Q M$ for some constant $c \neq 0$.) In view of Lemma $\mathrm{B}$, let $L(z)$ be an analytic branch of $\log w_{0}$ on an element $T_{1}$ of $F(a, b)$. We may assume that on $T_{1},|z|>e_{p}(0)$ (see $\$ 2(b)$ ), and we will use the notation $w_{0}^{a}$ for $\exp (\alpha L)$ if $\alpha$ is a real number. We now show that there is an element $T_{2}$ of $F(a, b)$, contained in $T_{1}$, such that,

$$
w_{0}^{\prime} \text { is nowhere zero on } T_{2} \text {. }
$$

The proof of this assertion is almost identical to the proof of [2, statement (30), p. 271], and so we simply sketch it. $w_{0}$ satisfies an equation of the form (14), whose coefficients $g_{k j}$ belong to a field of rank $p$. Let $t=\min \left\{j: g_{k j} \not 0\right.$ for some $k\}$, so in view of (57), $w_{0}$ satisfies the equation

$$
\sum\left\{g_{k j} w^{k}\left(w^{\prime}\right)^{j-t}: j \geq t\right\}=0 .
$$

Letting $\lambda$ and $\sigma$ be respectively the minimum and maximum of $\left\{k: g_{k t} \not \equiv 0\right\}$, we consider the algebraic polynomial $\Phi(v)=\Sigma_{k=\lambda}^{\sigma} g_{k t} v^{k}$. Using [9, Theorem II] (as in Lemma $C$ ), there is a logarithmic field of rank $p$ in which $\Phi$ factors completely. 
Letting $B_{1}, \cdots, B_{q}$ be the distinct nonzero roots of $\Phi$, it follows from (57) that there is an element $T_{3}$ of $F(a, b)$ on which $g_{\sigma t}$ is nowhere zero, and $\left|1-\left(B_{j}(z) / w_{0}(z)\right)\right| \geq 1 / 2$ for $j=1, \cdots, q$. Letting $T_{2}$ be an element of $F(a, b)$ contained in $T_{1}$ and $T_{3}$, it is easy to see that (58) holds.

In view of (58), we will let $L_{1}(z)$ be a fixed analytic branch of $\log \theta w_{0}$ in $T_{2}$, and we will use the notation, $\left(\theta w_{0}\right)^{\alpha}$ for $\exp \left(\alpha L_{1}\right)$ if $\alpha$ is a real number.

We return now to inequality (51) and let $\beta$ be as in (56). We define

$$
\gamma=\max \{\beta(k+j)-j:(k, j) \in D\}
$$

$$
\Delta=\{(k, j):(k, j) \in D \text { and } \beta(k+j)-j=\gamma\} .
$$

From (56) and $\S 2(d)$, we have

$$
\theta w_{0} \ll\left(\log _{p+1} z\right)^{\beta+\epsilon-1} \text { for all } \epsilon>0 \text {, over } F(a, b) \text {. }
$$

By choosing the arbitrary $\epsilon>0$ in (56) and (61) sufficiently small, it is clear from $(51)$ and the definition of $\Delta$, that there is an $\epsilon_{3}>0$ such that

$$
\sum_{(k, j) \epsilon \Delta} c_{k j} w_{0}^{k}\left(\theta w_{0}\right)^{j}=O\left(\left(\log _{p+1} z\right)^{\gamma-\epsilon_{3}}\right) \text { over } F(a, b) .
$$

Clearly, from $(60)$, if $(k, j)$ and $\left(k_{1}, j_{1}\right)$ are distinct elements of $\Delta$, then $k+j \neq$ $k_{1}+j_{1}$. Let $\left(k_{1}, j_{1}\right), \cdots,\left(k_{n}, j_{n}\right)$ be the distinct elements of $\Delta$, so arranged that if $q_{i}=k_{i}+j_{i}$ then $q_{1}<q_{2}<\cdots<q_{n}$. Letting $c_{i}$ denote $c_{k_{i} j_{i}}$ (62) may be written

$$
\sum_{i=1}^{n} c_{i} w_{0}^{q_{i}-j_{i}}\left(\theta w_{0}\right)^{j_{i}}=O\left(\left(\log _{p+1} z\right)^{\gamma-\epsilon_{3}}\right) \text { over } F(a, b) .
$$

At this point, we observe that

(64) If $\Delta$ has only one element (i.e. $n=1$ ), then $j_{1}>0$. To see this, we note that if $n=1$ but $j_{1}=0$, then from (63) and the fact that $\gamma=\beta q_{1}$, it would follow that by choosing $\epsilon$ in (56) sufficiently small, we would obtain $c_{1} \rightarrow 0$ contradicting the fact that $c_{1}$ is a nonzero constant.

Since $j_{i}=\beta q_{i}-\gamma$, we may write (63) as

$$
w_{0}^{\gamma}\left(\theta w_{0}\right)-\gamma_{E}\left(v_{0}\right)=O\left(\left(\log _{p+1} z\right)^{\left.\gamma-\epsilon_{3}\right)},\right.
$$

where

$$
v_{0}=w_{0}^{1-\beta}\left(\theta w_{0}\right)^{\beta} \text { and } E(v)=\sum_{i=1}^{n} c_{i} v^{q_{i}}
$$

Clearly $v_{0}=w_{0}$ if $\beta=0$. If $\beta \neq 0$, set 


$$
g(z)=((1 / \beta)-1) L(z)+L_{1}(z) \text {, so } v_{0}=\exp (\beta g),
$$

where $L$ and $L_{1}$ are the fixed branches of $\log w_{0}$ and $\log \theta w_{0}$ respectively. Now set

$$
V=\beta w_{0}^{1 / \beta}=\beta \exp (L / \beta)
$$

Thus,

$$
\theta V=\exp (g(z))=w_{0}^{(1 / \beta)-1}\left(\theta w_{0}\right), \quad \text { if } \beta \neq 0 .
$$

Finally, let $\mu_{1}, \cdots, \mu_{t}$ be the distinct nonzero roots of $E$ (if any), with $\mu_{j}$ of multiplicity $m_{j} \cdot$ Let $\mu_{0}=0$ and $m_{0}=q_{1}$.

As in [2, pp. 274-279], we will distinguish the two cases $\gamma \geq 0$ and $\gamma<0$, and in each case, we will distinguish subcases according to the sign of $\beta$. The proof that each case leads to a contradiction is almost identical to the corresponding proof in [2], and hence we will simply sketch these proofs.

Case I. $y \geq 0$. By choosing $\epsilon$ sufficiently small in (56) and (61), we have from (65) that $E\left(v_{0}\right)=O\left(\left(\log _{p+1} z\right)^{-\epsilon_{4}}\right)$ for some $\epsilon_{4}>0$. Thus from Appendix Lemma 1 , there is an index $k$, such that

$$
v_{0}-\mu_{k}=O\left(\left(\log _{p+1} z\right)^{-\epsilon_{5}}\right), \text { for some } \epsilon_{5}>0 \text {. }
$$

If $\beta=0$, then (70) is impossible by (57) (if $\mu_{k} \neq 0$ ) and by (56) (if $\mu_{k}=0$ ). If $\beta \notin 0$, then $\beta>0$ since $\beta \geq i_{n} / q_{n}$. If $\mu_{k}=0$ in (70), then from (69), $\theta V=$ $O\left(\left(\log _{p+1} z\right)^{-\epsilon s / \beta}\right)$. Applying Appendix Lemma 3 and (68), we obtain a contradiction of (56). The other possibility in (70) is that $\mu_{k} \neq 0$. We now argue as on p. 275 of [2]. Setting $\phi=v_{0}-\mu_{k}$ and choosing a branch $A(z)$ of $\log \left(\mu_{k}+z\right)$ on $|z|<\left|\mu_{k}\right|$, we set $W=\exp (A / \beta)$. Using the power series expansion for $W,(70)$, (67), (69), it follows as on [2, p. 275], that there is a nonzero complex number $\lambda_{4}$ such that if $U=V-\lambda_{4} \log _{p+1} z$, then $\theta U=O\left(\left(\log _{p+1} z\right)^{-\epsilon}\right)$. From Appendix Lemma 3, we obtain $V /\left(\lambda_{4} \log _{p+1} z\right) \rightarrow 1$. Applying Appendix Lemma 4, we obtain a contradiction of (57), so Case $I$ is complete.

Case II. $\gamma<0$. We let $\sigma=-\gamma$ so $\sigma>0$. From (66), we observe that

$$
\left|v_{0} / w_{0}\right|=\left|\theta w_{0} / w_{0}\right|^{\beta}
$$

We distinguish the three subcases $\beta>0, \beta<0$ and $\beta=0$.

Subcase A. $\beta>0$. From (65) and (71), it follows that by choosing $\epsilon$ in (56) sufficiently small, we obtain, $\left|v_{0}\right|^{\sigma / \beta}\left|E\left(v_{0}\right)\right| \leq K\left|\log _{p+1} z\right|^{-\epsilon} 4$ on some element of $F(a, b)$, where $K$ and $\epsilon_{4}$ are positive constants. Since $q_{1}+(\sigma / \beta)>0$, it thus follows from Appendix Lemma 1, that (70) holds for some root $\mu_{k}$. Since $\beta>0$ 
here, the proof proceeds exactly as in Case I to show that both $\mu_{k}=0$ and $\mu_{k} \neq 0$ are impossible.

Subcase B. $\beta<0$. Set $\eta=-\beta$ so $\eta>0$. From (65) and (71), it follows by choosing $\epsilon$ sufficiently small in (56), that $(65)$ may be written

$$
\left|v_{0}(z)\right|^{-\lambda}\left|v_{0}(z)-\mu_{1}\right|^{m} \cdots\left|v_{0}(z)-\mu_{t}\right|^{m}<\left|\log _{p+1} z\right|^{-\epsilon_{4}},
$$

on some element $S_{2}$ of $F(a, b)$, where $\epsilon_{4}>0$ and where $\lambda=(\sigma / \eta)-q_{1}$. (From (64) and (60), $\lambda>0$.) As in [2, pp. 276-277], we choose positive numbers, $\delta, K^{*}$ and $K_{0}$ such that, $\left|\mu_{i}-\mu_{j}\right| \geq \delta$ if $i \neq j,\left|\mu_{j}\right| \geq \delta$ for each $j \geq 1,\left|\mu_{j}\right| \leq K^{*}$ for each $j \geq 1, K_{0}>3 /(2 \delta)$ and $2 K_{0}^{2}-K^{*} K_{0}-1>0$. Let $S_{3}$ be an element of $F(a, b)$, contained in $S_{2}$, on which

$$
\left|\log _{p+1} z\right|^{-\epsilon_{4}}<\left(1 / 2 K_{0}\right)^{\lambda+m_{1}+\cdots+m_{t}}
$$

There are now two possibilities.

Possibility 1. There exists a point $z_{1}$ in $S_{3}$ and an index $k, 1 \leq k \leq t$, such that $\left|v_{0}\left(z_{1}\right)-\mu_{k}\right|<1 / 2 K_{0}$. In view of (72) and (73), it follows exactly as on p. 277 of [2] that for this index $k,(70)$ holds for some $\epsilon_{s}>0$. Since $\mu_{k} \neq 0$ and $\beta \neq 0$, the proof now proceeds exactly as in the last part of Case $I$, to obtain a contradiction of (57).

Possibility 2. If Possibility 1 fails, then from (72), it follows that $\left|v_{0}(z)\right|^{-\lambda} \leq K_{1}\left|\log _{p+1} z\right|^{-\epsilon_{4}}$ on $S_{3}$ for some $K_{1}>0$. Since $\lambda>0$ and $\beta<0$, it now follows from $(66)$ and $(69)$ that $\theta V=O\left(\left(\log _{p+1} z\right)^{-\epsilon_{5}}\right)$ for some $\epsilon_{s}>0$. Applying Appendix Lemma 3, and using (68) (noting that $\beta<0$ ), we obtain a contradiction of (56).

Subcase C. $\beta=0$, so $v_{0}=w_{0}$. From the comparability of $w_{0}$ with all monomials of rank $\leq p+1$, together with assumption (57), it follows that either $w_{0} \gg 1$ or $w_{0} \ll 1$.

Case (a), $w_{0} \gg 1$. Since $v_{0}=w_{0}$, it follows easily from (65) (and the fact that $\sigma=j_{n}$ since $\left.\beta=0\right)$, that if we let $U=w_{0}^{\zeta}+1 /(\zeta+1)$, where $\zeta=\left(q_{n}-\sigma\right) / \sigma$, then

$$
\theta U=O\left(\left(\log _{p+1} z\right)^{-1-\epsilon_{3} / \sigma}\right) \text { and } \zeta \geq 0
$$

From Appendix Lemma 2, it follows that if $z_{0}$ is a fixed point with $\arg z_{0}=$ $(a+b) / 2$ and of sufficiently large modulus, then $\left|U\left(R z_{0}\right)\right|$ is bounded as a function of $R$ on $[1,+\infty)$. Since $\zeta \geq 0$, this clearly contradicts the assumption $w_{0} \gg 1$ over $F(a, b)$.

Case (b). $w_{0} \ll 1$. In this case it follows from (65) that if $U=w_{0}^{\zeta+1} /(\zeta+1)$, 
where $\zeta=\left(q_{1}-\sigma\right) / \sigma$, then (74) again holds. Thus there exist $K>0$ and an element $T$ in $F(a, b)$ such that

$$
|\theta U| \leq K\left|\log _{p+1} z\right|^{-1-\epsilon_{3} / \sigma} \text { on } T,
$$

and $|z|>e_{p+1}(0)$ for all $z$ in $T$. Now let $z_{1}$ be any point in $T$ with $\arg z_{1}=$ $(a+b) / 2$. By Appendix Lemma 2,

$$
\left|U\left(R z_{1}\right)-U\left(z_{1}\right)\right| \leq\left(K \sigma / \epsilon_{3}\right)\left(\log _{p+1}\left|z_{1}\right|\right)^{-\epsilon_{3} / \sigma} \text { for all } R \geq 1 .
$$

But since $w_{0} \ll 1$, clearly $\left|U\left(R z_{1}\right)\right| \rightarrow 0$ as $R \rightarrow+\infty$, so $\left|U\left(z_{1}\right)\right| \leq$ $\left(K \sigma / \epsilon_{3}\right)\left(\log _{p+1}\left|z_{1}\right|\right)^{-\epsilon_{3} / \sigma}$. Since $\zeta \geq 0$, it follows that if we set $\epsilon_{4}=\epsilon_{3} / \sigma(\zeta+1)$, then $\epsilon_{4}>0$ and for any point $z_{1}$ in $T$ with $\arg z_{1}=(a+b) / 2$, we have

$$
\left|w_{0}\left(z_{1}\right)\right| \leq\left((\zeta+1) K \sigma / \epsilon_{3}\right)^{1 /(\zeta+1)}\left(\log _{p+1}\left|z_{1}\right|\right)^{-\epsilon_{4}} \text {. }
$$

But $\beta=0$, so by $(56), w_{0} \gg\left(\log _{p+1} z\right)^{-\epsilon_{4} / 2}$. Thus on some element $T_{0}$ of $F(a, b)$, contained in $T$, we have

$$
\left|w_{0}(z)\right|>\left|\log _{p+1} z\right|^{-\epsilon_{4} / 2}
$$

Now $p$ is a fixed positive integer, and it is easy to prove (by induction on $p$ ) that there is a positive number $R(p)$ such that

$$
\left|\log _{p+1} z\right| \leq 2\left(\log _{p+1}|z|\right) \text { for }|\arg z|<\pi \text { and }|z|>R(p) \text {. }
$$

Since $T_{0}$ contains points $z_{1}$, with $\arg z_{1}=(a+b) / 2$ and $\left|z_{1}\right|$ arbitrarily large, it is clear that (77), (78) and (79) would yield the fact that for such points $z_{1}$, $\left(\log _{p+1}\left|z_{1}\right|\right)^{\epsilon / 2}$ is bounded which is of course absurd. Thus Case (b) has also led to a contradiction.

Hence every case has led to a contradiction and this proves Lemma G.

14. Proof of the main result ( $\$ 3)$. The first statement of the conclusion follows from Lemma $B(\$ 7)$ and the remark following it. If $p=0$, the rest of the conclusion follows from $\left[2, \S_{3}\right]$, so we may assume $p \geq 1$. By Lemma $A(\S 5), y_{0}$ possesses a p-exponent sequence of some length $q$. If $q=0$, the rest of the conclusion follows from $[1, \S 3]$ (i.e. (B) of the conclusion holds), so we may assume $q \geq 1$. If the p-exponent sequence is nonlogarithmic, the rest of the conclusion follows from Lemma D ( $\$ 9)$ (i.e. (B) of the conclusion holds). If the p-exponent sequence is logarithmic, the rest of the conclusion follows from Lemma $G(\$ 13)$ (i.e. part (A) of the conclusion holds). 
15. Remarks. (1) We consider three types of functions / which are analytic in an element of $F(a, b)$. (i) $f$ nowhere zero and $f=o\left(z^{-a}\right)$ for all $a>0$ over $F(a, b)$; (ii) for all $a>0, z^{-a} f \rightarrow \infty$ over $F(a, b)$; (iii) for some logarithmic monomial $M, f \sim M$ over $F(a, b)$. We show that each such function is power-comparable with all logarithmic monomials over $F(a, b)$. For (i), let $L$ be an analytic branch of $\log f$ and let $n$ be a positive integer. Since $z^{a_{n}} f \rightarrow 0$, clearly, $z^{a} e^{L / n} \rightarrow 0$ over $F(a, b)$ for all $a>0$. By $[3, \$ 7]$, it follows that $e^{L / n} \ll z^{-a}$ for all $a>0$ over $F(a, b)$, proving the conclusion. Case (ii) follows from Case (i). For (iii), let $M$ have the form (2). Clearly, there is an analytic branch $L$ of $\log f$ in some element of $F(a, b)$, and let $n$ be a positive integer. The conclusion will easily follow if we can show

$$
z^{-a_{0} / n}(\log z)^{-a_{1} / n} \ldots\left(\log _{p} z\right)^{-a_{p} / n} e^{L / n} \approx 1 \text { over } F(a, b) .
$$

To prove (80), denote by $E$ the left side of (80). Since $f \sim M$, clearly $E^{n} \sim K$ where $K$ is the nonzero constant in (2). By Appendix Lemma 1, there is an nth root of $K$, call it $\lambda$, such that $E \rightarrow \lambda$ over $F(a, b)$. Let $G=E-\lambda$, so $G \rightarrow 0$. Let $E^{n}=K+H$, where $H \ll 1$. Substituting $(G+\lambda)^{n}$ into this relation for $E^{n}$ and expanding, it follows from $[7, \S 33]$ that $G \ll 1$. Thus $E \sim \lambda$ and $(80)$ is proved.

(2) We construct an example of a function, analytic in an element of $F(-\pi, \pi)$, which over $F(-\pi, \pi)$, is power-comparable with all logarithmic monomials, but which is neither $\sim$ to a logarithmic monomial nor of the form $\exp \int N(z)(1+o(1))$, where $N$ is a logarithmic monomial. To proceed, let $T$ be an element of $F(-\pi, \pi)$ on which $|z|>e^{\pi}$, and let $z_{0} \in T$. Let $F(z)=\exp \int_{z_{0}}^{z}-\zeta^{-1}(\log \zeta) d \zeta$ on $T$. From $[3, \S 4]$, it follows that $F \ll z^{-a}$ for all $\alpha>0$ over $F(-\pi, \pi)$. Let $G(z)=$ $\exp \left(z^{i} \log z\right)$, where $i^{2}=-1$ and $z^{i}=\exp (i(\log z))$. Then $|G(z)| \leq|z|^{\lambda}$ on $T$, where $\lambda=2 e^{\pi}$. Hence if we set $H=F G$, then $H$ is nowhere zero on $T$ and $H=o\left(z^{-a}\right)$ over $F(-\pi, \pi)$ for all $\alpha>0$. From (i) in Remark (1) above, $H$ is power comparable with all logarithmic monomials and clearly $H$ is not $\sim$ to any logarithmic monomial over $F(-\pi, \pi)$. It remains to prove that $H$ is not of the form $\exp \int N(z)(1+E(z))$, where $N$ is a logarithmic monomial and $E \rightarrow 0$ over $F(-\pi, \pi)$. If we assume the contrary for some monomial $N$, we would have

$$
i z^{i}=1-\left(z^{i} / \log z\right)+z(\log z)^{-1} N(z)(1+E(z)) .
$$

Since one of the relations, $N \gg z^{-1}(\log z), N \approx z^{-1}(\log z), N \ll z^{-1}(\log z)$, must hold over $F(-\pi, \pi)$, it would follow from (81) that $z^{i} \rightarrow \infty$ or $z^{i} \rightarrow c$ for some constant $c$, over $F(-\pi, \pi)$, which is absurd.

(3) In [2], the printer omitted the constant $c_{n-j, j}$ which should appear 
immediately after the summation signs in (15) and (19) on p. 269 of [2]. In addition, $w_{j}$ should be $w^{j}$ in (19).

\section{Appendix.}

Lemma 1. Let $\lambda_{1}, \cdots, \lambda_{q}$ be $q \geq 1$ distinct complex numbers and let $m_{1}, \cdots$, $m_{q}$ be positive real numbers. Let $\phi(z)$ and $\nu_{0}(z)$ be analytic functions in an element $T$ of $F(a, b)$, sucb that $\phi \rightarrow 0$ over $F(a, b)$ and sucb that for some $K_{1}>0$,

$$
\left|v_{0}(z)-\lambda_{1}\right|^{m} \cdots\left|v_{0}(z)-\lambda_{q}\right|^{m} q \leq K_{1}|\phi(z)| \text { on } T \text {. }
$$

Then there exist $K_{2}>0, T_{1} \in F(a, b)$ and an index $t, 1 \leq t \leq q$, sucb that $\mid v_{0}(z)-$ $\left.\lambda_{t}\left|\leq K_{2}\right| \phi(z)\right|^{1 / m_{t}}$ on $T_{1}$.

Proof. The proof is identical to the proof of [2, Appendix Lemma 1, p. 281] with $\phi(z)$ replacing $(\log z)^{-\epsilon}$.

Lemma 2. Let $V(z)$ be analytic in an element $T$ of $F(a, b)$, and let $\epsilon>0$, $K>0$ and $q \geq 1$ be such that on $T$,

$$
\left|V^{\prime}(z)\right| \leq K|z|^{-1} \cdots\left|\log _{q-1} z\right|^{-1}\left|\log _{q} z\right|^{-1-\epsilon} .
$$

Then for any point $z_{1}$ in $T$ with $\arg z_{1}=(a+b) / 2$ and $\left|z_{1}\right|>e_{q}(0)$, we bave $\left|V\left(R z_{1}\right)-V\left(z_{1}\right)\right| \leq(K / \epsilon)\left(\log _{q}\left|z_{1}\right|\right)^{-\epsilon}$ for all $R \geq 1$.

Proof. The proof follows easily from the fact that $\left|\log _{j} r z\right| \geq \log _{j}\left|r z{ }_{1}\right|>0$ if $r \geq 1$ and $j \leq q$, and the fundamental theorem of calculus.

Lemma 3. Let $V(z)$ be analytic in an element $T$ of $F(a, b)$, where $T$ bas the property that $|z|>e_{p}(0)$ for all $z$ in $T$, and let $\epsilon>0$ and $K>0$ be such that on $T$, we bave

$$
\left|V^{\prime}(z)\right| \leq K|z|^{-1}|\log z|^{-1} \cdots\left|\log _{p} z\right|^{-1}\left|\log _{p+1} z\right|^{-\epsilon}
$$

Then there exists a number $\epsilon^{\prime}$, with $0<\epsilon^{\prime}<1$, such that $V=O\left(\left(\log _{p+1} z\right)^{1-\epsilon^{\prime}}\right)$ over $F(a, b)$.

Proof. The proof follows exactly along the lines of [2, Appendix Lemma 3, p. 282], where it was proved for the case $p=0$.

Lemma 4. Let $w_{0}(z)$ be analytic and nowbere zero in an element of $F(a, b)$, and let $L(z)$ be a branch of $\log w_{0}$. Let $V(z)=\beta(\exp (L(z) / \beta))$, where $\beta$ is a nonzero real number. Then if for some nonzero constant $c_{1}$ we have $V(z) / c_{1} \log _{p+1} z \rightarrow 1$ over $F(a, b)$, then there is a nonzero constant $c_{2}$ sucb that $w_{0}(z) / c_{2}\left(\log _{p+1} z\right)^{\beta} \rightarrow 1$ over $F(a, b)$. 
Proof. The proof follows exactly along the lines of [2, Appendix Lemma 4, p. 283], where it was proved for the case $p=0$.

Lemma 5. Let $q$ be a positive integer, and let $w_{0}(z)$ be a meromorphic function in an element of $F(a, b)$, which is power-comparable with all logaritbmic monomials of rank $\leq q$ over $F(a, b)$. Suppose that for every $a>0$, it is true that $w_{0} \gg\left(\log _{q} z\right)^{a}$ over $F(a, b)$. Then if $j$ and $m$ are nonnegative integers witb $j<m$, we bave

$$
\left(w_{0}^{\prime}\right)^{j} / w_{0}^{m}=o\left(z^{-j(\log z)^{-j}} \ldots\left(\log _{q-1} z\right)^{-j\left(\log _{q} z\right)^{-a}}\right)
$$

for all $a>0$ over $F(a, b)$.

Proof. For $j=0$, the result clearly holds, so we may assume $j \geq 1$. Let $L$ be a branch of $\log w_{0}$, and let $\epsilon=(m-j) / j$. Thus $\epsilon>0$, and

$$
\left(w_{0}^{\prime}\right)^{j} / w_{0}^{m}=\left(w_{0}^{\prime} / e^{(1+\epsilon) L}\right)^{j} .
$$

Let $n$ be a positive integer such that $1 / n<\epsilon$, and let $\alpha>0$. Choose $\lambda>0$ so that $a<j(\lambda+1)$. From the hypothesis, clearly $e^{L / n}\left(\log _{a} z\right)^{-\lambda} \rightarrow \infty$, and since $e^{L / n}$ is comparable with $\left(\log _{q} z\right){ }^{\lambda}$ we must have $e^{L / n} \gg\left(\log _{q} z\right)^{\lambda}$ over $F(a, b)$. Thus if $f=\left(\log _{q} z\right)^{\lambda} e^{-L / n}$, then $f \ll 1$ over $F(a, b)$. Hence (see $\left.\$ 2(\mathrm{~d})\right)$, we have $\theta_{q+1} f \ll 1$ over $F(a, b)$. Calculating $\theta_{q+1} f$, and using the fact that each of the functions, $\lambda f$, $\exp (((1 / n)-\epsilon) L),\left(\log _{q} z\right)^{(a / j)-(\lambda+1)}$ tends to zero over $F(a, b)$, it easily follows that

$$
e^{-(1+\epsilon) L}\left(\theta_{q} w_{0}\right)\left(\log _{q} z\right)^{a / j} \rightarrow 0 \text { over } F(a, b) .
$$

In view of (A5), we thus obtain (A4).

\section{BIBLIOGRAPHY}

1. S. Bank, A representation theorem for large and small analytic solutions of algebraic differential equations in sectors, Trans. Amer. Math. Soc. 159 (1971), 293-305. MR 44 \#505.

2. - On the asymptotic representation of analytic solutions of first-order algebraic differential equations in sectors, Trans. Amer. Math. Soc. 176 (1973), 263-283.

3. - On solutions having large rate of growth for non-linear differential equations in the complex domain, J. Math. Anal. Appl. 22 (1968), 129-143. MR 40 \#5945.

4. - On the instability theory of differential polynomials, Ann. Mato Pura Appl. (4) 74 (1966), 83-111. MR 34 \#4623.

5. E. W. Chamberlain, Families of principal solutions of ordinary differential equations, Trans. Amer. Math. Soc. 107 (1963), 261-272. MR $26 \# 6470$.

6. - The univalence of functions asymptotic to nonconstant logarithmic monomials, Proc. Amer. Math. Soc. 17 (1966), 302-309. MR 32 \#7724.

7. W. Strodt, Contributions to the asymptotic theory of ordinary differential equations in the complex domain, Mem. Amer. Math. Soc. No. 13 (1954). MR 16, 702. 
8. W. Strodt, Principal solutions of ordinary differential equations in the complex domain, Mem. Amer. Math. Soc. No. 26 (1957). MR 19, 1177.

9. - On the algebraic closure of certain partially ordered fields, Trans. Amer. Math. Soc. 105 (1962), 29-250. MR 25 \#3934.

10. W. Strodt and R. K. Wright, Asymptotic behavior of solutions and adjunction fields for nonlinear first order differential equations, Mem. Amer. Math. Soc. No. 109 (1971). MR 44 \#1884.

DEPARTMENT OF MATHEMATICS, UNIVERSITY OF ILLINOIS, URBANA, ILLINOIS 61801 